International Journal of Pure and Applied Mathematics

Volume 100 No. 4 2015, 475-478

ISSN: 1311-8080 (printed version); ISSN: 1314-3395 (on-line version)

url: http://www.ijpam.eu

doi: http://dx.doi.org/10.12732/ijpam.v100i4.6

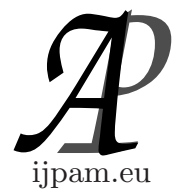

\title{
ON THE HYPERCYCLICITY AND WEIGHTED COMPOSITION OPERATORS ON BANACH FUNCTION SPACES
}

\author{
Bahmann Yousefi ${ }^{1}$, Fariba Ershad $^{2 \S}$ \\ ${ }^{1,2}$ Department of Mathematics \\ Payame Noor University \\ P.O. Box 19395-3697, Tehran IRAN
}

\begin{abstract}
In this paper we investigate the hypercyclicity of adjoint of a special weighted composition operators on a Banach function space.
\end{abstract}

AMS Subject Classification: 47B37, 47B38

Key Words: weighted composition operator, hypercyclicity

\section{Introduction}

Let $\mathcal{B}(X)$ denotes the set of all bounded linear operators on a Banach space $X$. An operator $T \in \mathcal{B}(X)$ is said to be hypercyclic if there exists a vector $x \in X$ for which the orbit $\operatorname{Orb}(T, x)=\left\{T^{n} x: n \in \mathbb{N}\right.$ is dense in $X$ and in this case we refer to $x$ as a hypercyclic vector for $T$. The holomorphic self maps of the open unit disk $U$ are divided into classes of elliptic and non-elliptic. The elliptic type is an automorphism and has a fixed point in $U$. It is well known that this map is conjugate to a rotation $z \rightarrow \lambda z$ for some complex number $\lambda$ with $|\lambda|=1$. The maps of that are not elliptic are called of non-elliptic type. The iterate of a non-elliptic map can be characterized by the Grand Iteration Theorem ([3, p. $78])$. By $\psi^{\prime}(w)$ we denote the angular derivative of $\psi$ at $w \in \partial U$. Note that if $w \in U$, then $\psi^{\prime}(w)$ has the natural meaning of derivative. Also, by $\psi_{n}$ we mean the nth iteration of the function $\psi$.

Received: December 24, 2014

(C) 2015 Academic Publications, Ltd.

$\S$ Correspondence author url: www.acadpubl.eu 
Proposition 1.1. (see [6]) Suppose $\psi$ is a holomorphic self-map of $U$ that is not an elliptic automorphism. If $\psi$ has a fixed point $w \in U$, then $\psi_{n} \rightarrow w$ uniformly on compact subsets of $U$ and $\left|\psi^{\prime}(w)\right|<1$.

The unique attracting point $w$ in the above proposition is called the DenjoyWolff point of $\psi$.

Consider the weighted composition operator $C_{\varphi, \psi}$ on a Banach space $X$ of analytic functions defined by

$$
C_{\varphi, \psi} f=\varphi \cdot f \circ \psi \quad(f \in X) .
$$

We will investigate the hypercyclicity of the operator $C_{\varphi, \psi}^{*}$. For some sources we refer to [1-4].

\section{Main Results}

Let $X$ be a Banach space of analytic functions on the open unit disk $U$. For each $\lambda \in U$, the evaluation function $e_{\lambda}: X \rightarrow C$ is defined by $e_{\lambda}(f)=f(\lambda)$, $f \in X$. A complex valued function $\varphi$ on $U$ for which $\varphi X \subseteq X$ is called a multiplier of $X$. The set of all multipliers of $X$ is denoted by $M(X)$ and we have $M(X) \subseteq H^{\infty}(U)$.

It is well known that if the adjoint of a continuous operator $T$ on a Banach space has an eigenvector, then $T^{*}$ fails to be hypercyclic (see [2]).

Lemma 2.1. Let $X$ be a separable Banach space of analytic functions on the open unit disk $U$ such that $1 \in X$, and for each $\lambda \in U, e_{\lambda} \in X^{*}$. Also, let $z \in M(X)$ and the map $\varphi \rightarrow M_{\varphi}$ from $M(X)$ into $\mathcal{B}(X)$ is an isometry. Then $M(X)=H^{\infty}(U) \subset X$.

Proof. Let $f \in H^{\infty}(U)$. By the Farrell-Rubel-Shields Theorem [1, Theorem 5.1, p.151], there is a sequence $\left\{p_{n}\right\}_{n}$ of polynomials converging to $f$ such that for all $n,\left\|p_{n}\right\|_{U} \leq c_{0}$ for some $c_{0}>0$. So we obtain $\left\|M_{p_{n}}\right\|=\left\|p_{n}\right\|_{U} \leq c_{0}$ for all $n$. But ball $B(X)$ is compact in the weak operator topology and so by passing to a subsequence if necessary, we may assume that for some $A \in B(X)$, $M_{p_{n}} \longrightarrow A$ in the weak operator topology. Using the fact that $M_{p_{n}}^{*} \longrightarrow A^{*}$ in the weak operator topology and acting these operators on $e_{\lambda}$ we get $p_{n}(\lambda) e_{\lambda}=$ $M_{p_{n}}^{*} e_{\lambda} \longrightarrow A^{*} e_{\lambda}$ weakly. Since $p_{n}(\lambda) \longrightarrow f(\lambda)$ we see that $A^{*} e_{\lambda}=f(\lambda) e_{\lambda}$. Because the closed linear span of $\left\{e_{\lambda}: \lambda \in U\right\}$ is dense in $X^{*}$, we conclude that $A=M_{f}$ and this implies that $f \in M(X)$. Indeed $f \in X$, since $X$ contains the constant functions. 
Theorem 2.2. Let $X$ be a separable Banach space of analytic functions on the open unit disk $U$ such that $1 \in X$, and for each $\lambda \in U, e_{\lambda} \in X^{*}$. Also, let $z \in M(X)$ and the $\operatorname{map} \varphi \rightarrow M_{\varphi}$ from $M(X)$ into $\mathcal{B}(X)$ is an isometry. Let $0<\lambda<1$ and $\varphi \in M(X)$ be such that $\varphi(0) \neq 0$. Then $\left(C_{\varphi, \lambda z}\right)^{*}$ is not hypercyclic.

Proof. Since $\varphi$ is bounded, an application of Schwartz's Lemma shows that

$$
|\varphi(0)-\varphi(z)|<2|| \varphi \|_{U}|z|
$$

for all $z$ in $U$. But $\varphi(0) \neq 0$, thus

$$
\left|1-\frac{1}{\varphi(0)} \varphi(z)\right|<\frac{2|| \varphi \|_{U}}{|\varphi(0)|}|z| \quad(z \in U) .
$$

By substituting $\lambda^{i} z$ instead of $z$ in the above inequality, we get

$$
\left|1-\frac{1}{\varphi(0)} \varphi\left(\psi_{i}(z)\right)\right|<\frac{2|| \varphi \|_{U}}{|\varphi(0)|}|\lambda|^{i}|z| .
$$

This implies that

$$
\sum_{i=0}^{\infty}\left|1-\frac{1}{\varphi(0)} \varphi\left(\lambda^{i} z\right)\right|
$$

and consequently

$$
\prod_{i=0}^{\infty} \frac{1}{\varphi(0)} \varphi\left(\lambda^{i} z\right)
$$

converges uniformly on compact subsets of $U$. Set

$$
g(z)=\prod_{i=0}^{\infty} \frac{1}{\varphi(0)} \varphi\left(\lambda^{i} z\right)
$$

then $g$ is a nonzero holomorphic function on $U$. Also, note that

$$
|\varphi(z)|<2|| \varphi \|_{U}|z|+|\varphi(0)|
$$

for every $z \in U$. So it follows that if $z \in U$, then

$$
\left|\varphi\left(\lambda^{n} z\right)\right|<2|| \varphi \|_{U}|\lambda|^{n}|z|+|\varphi(0)| \text {. }
$$

But $\varphi(0) \neq 0$, thus

$$
\frac{\left|\varphi\left(\lambda^{n} z\right)\right|}{|\varphi(0)|}<2|| \varphi \|_{U}|\lambda|^{n} \frac{|z|}{|\varphi(0)|}+1
$$




$$
\begin{aligned}
& \leq \exp \left(2\|\varphi\|_{U}|\lambda|^{n} \frac{|z|}{|\varphi(0)|}\right) \\
& \leq \exp \left(\frac{2\|\varphi\|_{U}}{|\varphi(0)|}|\lambda|^{n}\right) .
\end{aligned}
$$

Therefore, we get

$$
\begin{aligned}
\prod_{n=0}^{\infty}\left|\frac{1}{\varphi(0)} \varphi\left(\lambda^{n} z\right)\right| & \leq \exp \left(\sum_{n=0}^{\infty}\left(\frac{2\|\varphi\|_{U}}{|\varphi(0)|}|\lambda|^{n}\right)\right. \\
& =\exp \left(\frac{2|| \varphi \|_{U}}{|\varphi(0)|} \frac{1}{1-|\lambda|}\right)
\end{aligned}
$$

for every $z \in U$. Hence, indeed $g$ belongs to $H^{\infty}(U)$ and so by Lemma 2.1, $g \in X$. But $\varphi \cdot g \circ \lambda z=\varphi(0) g$ and so $\varphi(0)$ is an eigenvalue for the operator $M_{\varphi} C_{\psi}$. Now since the operator $C_{\varphi, \lambda z}$ has a non-zero eigenvalue, then $\left(C_{\varphi, \lambda z}\right)^{*}$ fails to be hypercyclic. This completes the proof.

\section{References}

[1] . Gamelin, Uniform Algebra, New York (1984).

[1] G. Godefroy, J.H. Shapiro, Operators with dense invariant cyclic vector manifolds, J. Func. Anal., 98 (1991), 229-269.

[2] [3] J.H. Shapiro, Composition Operators and Classical Function Theory, Springer-Verlag, New York (1993).

[3] [4] B. Yousefi, H. Rezaei, Hypercyclic property of weighted composition operators, Proc. Amer. Math. Soc., 135, No. 10 (2007), 3263-3271. 